\title{
PREDICTION OF OUTSTANDING PAYMENTS IN A POISSON CLUSTER MODEL
}

\author{
ANDERS HEDEGAARD JESSEN, THOMAS MIKOSCH, \\ AND GENNADY SAMORODNITSKY
}

\begin{abstract}
We consider a simple Poisson cluster model for the payment numbers and the corresponding total payments for insurance claims arriving in a given year. Due to the Poisson structure one can give reasonably explicit expressions for the prediction of the payment numbers and total payments in future periods given the past observations of the payment numbers. One can also derive reasonably explicit expressions for the corresponding prediction errors. In the $(a, b)$-class of Panjer's claim size distributions, these expressions can be evaluated by simple recursive algorithms. We study the conditions under which the predictions are asymptotically linear as the number of past payments becomes large. We also demonstrate that, in other regimes, the prediction may be far from linear. For example, a staircase-like pattern may arise as well. We illustrate how the theory works on real-life data, also in comparison with the chain ladder method.
\end{abstract}

\section{INTRODUCTION}

Let $N_{k}$ be the number of payments for claims arriving in an insurance portfolio in the year 0 and being executed in the year $k \in\{0,1, \ldots\}$. Moreover, let $S_{k}$ be the corresponding total amount of the payments executed in year $k$. If one has observed the counts $N_{k}, k=0, \ldots, j$, for some $j \geq 0$, a major problem for an insurance company is to determine a reserve for the years $j+1, j+2, \ldots$. This amounts to predicting the pairs $\left(N_{j+\ell+1}, S_{j+\ell+1}\right)$ for $\ell=0,1, \ldots$. In this context natural estimators are given by the conditional expectations given the past values $N_{0}, \ldots, N_{j}$, i.e.,

(1.1) $\widehat{N}_{j+\ell+1}=E\left(N_{j+\ell+1} \mid \mathcal{F}_{j}\right) \quad$ and $\quad \widehat{S}_{j+\ell+1}=E\left(S_{j+\ell+1} \mid \mathcal{F}_{j}\right)$,

$\ell=0,1, \ldots$, where $\mathcal{F}_{j}=\sigma\left(N_{0}, \ldots, N_{j}\right), j=0,1, \ldots$ Assuming $\operatorname{var}\left(S_{j+\ell+1}\right)<$ $\infty, S=\widehat{S}_{j+\ell+1}$ is the a.s. unique minimizer of the mean square error $E\left(\left(S_{j+\ell+1}-S\right)^{2}\right)$ in the class of square integrable random variables $S$ which

1991 Mathematics Subject Classification. Primary 60K10, Secondary 60G25, 60G55.

Key words and phrases. Poisson cluster model, prediction, claims reserving, chain ladder method, Panjer distributions, $(a, b)$-class, recursive algorithm.

Thomas Mikosch's research is partly supported by the Danish Research Council (FNU) Grant 272-06-0442. Gennady Samorodnitsky's research is partly supported by the ARO grant W911NF-07-1-0078 at Cornell University and a Villum Kann Rasmussen Visiting Professor Grant at the University of Copenhagen. 
are measurable functions of $N_{0}, \ldots, N_{j}$, and a similar remark applies to $\widehat{N}_{j+\ell+1}$. Moreover, $s=\widehat{S}_{j+\ell+1}$ minimizes the conditional mean square error $E\left(\left(S_{j+\ell+1}-s\right)^{2} \mid \mathcal{F}_{j}\right)$.

One of the popular procedures in this context was suggested by Mack; see Mack [2, 3, 4] and Mack et al. [6]. In its simplest version, Mack's procedure declares the predictors $\widehat{S}_{j+\ell+1}$ and $\widehat{N}_{j+\ell+1}$ to be linear functions of $S_{0}+\cdots+S_{j}$ or $N_{j}=N_{0}+\cdots+N_{j}$, respectively. For example,

$$
\widehat{N}_{j+1}=\left(f_{j}-1\right)\left(N_{0}+\cdots+N_{j}\right), \quad j=0,1, \ldots,
$$

for constants $f_{j} \geq 1$.

Assume that one observes the run-off triangle

$$
\left(N_{i, i+k}, S_{i, i+k}\right), \quad i=1, \ldots, n, \quad k=0, \ldots, n-i,
$$

where $\left(\left(N_{i, i+k}, S_{i, i+k}\right)\right)_{k=0, \ldots, j-i}$ are the payment numbers and total payments for claims arriving in year $i$ and being executed in year $i+k$; one assumes that $\left(\left(N_{i, i+k}, S_{i, i+k}\right)\right)_{k=0,1, \ldots}$ are iid copies of $\left(\left(N_{k}, S_{k}\right)\right)_{k=0,1, \ldots}$. Here $n$ is the last year for which payments were observed. Mack's assumptions (1.2) give raise to constructing natural estimators $\widehat{f}_{j}$ of $f_{j}$ which are referred to as chain ladder estimators. Then, replacing the unknown parameters $f_{j}$ on the right-hand side of $(1.2)$ by their estimators $\widehat{f}_{j}$, one obtains a predictor of $N_{j+1}$. In Section 5 we we will introduce the chain ladder estimators and compare the performance of the corresponding predictors with those proposed in this paper.

Mack's procedure (1.2) does not determine the dynamics of a particular stochastic process. For example, one cannot simulate a process $\left(N_{j}\right)_{j=0,1, \ldots}$ from (1.2). Moreover, the linearity of the estimator (1.2) is a simplification which is hard to reconcile with natural stochastic models for the count process.

In this paper, we consider a simple stochastic process model for the counts $N_{j}, j=0,1, \ldots$, and the corresponding payments $S_{j}, j=0,1, \ldots$ The model is given by the following conditions which we assume throughout this paper.

The model. Let $M$ be the number of claims arriving in a given year with distribution

$$
q_{m}=P(M=m), \quad m=0,1, \ldots
$$

The $m$ th claim causes a stream of $K_{m}$ payments from the insurer to the insured through the next years. We assume that the $k$ th of these payments is executed in the year $Y_{m k}$. We further assume that $\left(K_{m}\right)$ is an iid sequence of $\operatorname{Poisson}(\mu)$ distributed random variables and that $\left(Y_{m k}\right)_{m, k=1,2, \ldots}$ constitutes an iid family with common distribution

$$
p_{j}=P\left(Y_{11}=j\right), \quad j=0,1, \ldots .
$$


Finally, assume that $M,\left(K_{m}\right)$ and $\left(Y_{m k}\right)$ are independent. Write

$$
N_{j}=\sum_{m=1}^{M} \sum_{k=1}^{K_{m}} I_{\left\{Y_{m k}=j\right\}}, \quad j=0,1, \ldots,
$$

i.e., $N_{j}$ is the number of payments for claims arriving in a given year and being executed in year $j$. Assume further that $\left(X_{m k}\right)_{m, k=1,2, \ldots}$ is an iid family of non-negative random variables independent of $M,\left(K_{m}\right)$ and $\left(Y_{m k}\right)$. We interpret $X_{m k}$ as the $k$ th payment for the $m$ th claim. Then

$$
S_{j}=\sum_{m=1}^{M} \sum_{k=1}^{K_{m}} X_{m k} I_{\left\{Y_{m k}=j\right\}}, \quad j=0,1, \ldots,
$$

are the total payments for the claims arriving in year 0 and being executed in year $j$.

Both processes $\left(N_{j}\right)$ and $\left(S_{j}\right)$ can easily be simulated. It is our aim to show that the predictors (1.1) and their errors can be calculated explicitly and are easily derived by numerical methods for certain special cases of the distribution of $M$. The expressions for the predictor of $N_{j+1}$ are highly non-linear functions of $N_{0}+\cdots+N_{j}$, in contrast to Mack's procedure (1.2). However, under some condition on the distribution of $M$ these predictors are asymptotically linear functions of $N_{0}+\cdots+N_{j}$ if the latter quantity increases to infinity. In other situations, the predictors stay non-linear even in the limit.

The paper is organized as follows. We start in Section 2 by giving the relevant formulas for the predictors of $S_{j+\ell+1}, \ell=0,1, \ldots$. Since $S_{j}=N_{j}$ if $X_{m i}=1$ for all $m, i$, the prediction of $N_{j+\ell+1}, \ell=0,1, \ldots$, is a special case. We also determine the prediction errors. The predictors and conditional prediction errors involve certain derivatives of the Laplace-Stieltjes transform of $M$. In general, these derivatives are difficult to obtain. However, in the $(a, b)$-class of Panjer distributions, including the Poisson, binomial and negative binomial distributions, there exist simple recursive algorithms for calculating these derivatives; see Section 3. In Section 4 we study the asymptotic behavior of the predictors as the number of the previously observed payments grows. In particular, we give conditions under which the predictors $\widehat{S}_{j+\ell+1}$ are asymptotically linear functions of $N_{0}+\cdots+N_{j}$. We also consider other situations, where different asymptotic patterns of the behavior of the predictors arise. An interesting feature is the staircase-like pattern discussed in that section. In Section 5 we apply our predictors to a non-life insurance data set. We compare the performance of these predictors with the corresponding ones based on chain ladder estimation.

\section{THE PREDICTION PROBLEM}

We intend to predict the future numbers of payments $N_{j+\ell+1}$ and the corresponding total claim amounts $S_{j+\ell+1}, \ell=0,1, \ldots$, given the past payment numbers $N_{0}, \ldots, N_{j}$. This means we will calculate the predictors $\widehat{S}_{j+\ell+1}$ and 
$\widehat{N}_{j+\ell+1}$ in (1.1) provided these quantities are well-defined. Since we always assume the conditions of the model introduced in Section 1, the independence of $\left(X_{m i}\right)$ and the rest of the random ingredients in the model implies that

$$
\widehat{S}_{j+\ell+1}=E X_{11} E\left(N_{j+\ell+1} \mid \mathcal{F}_{j}\right)=E X_{11} \widehat{N}_{j+\ell+1},
$$

$\ell=0,1, \ldots$. Therefore the prediction problem for $S_{j+\ell+1}$ reduces to the one for $N_{j+\ell+1}$. Conversely, if $X_{m i}=1$ a.s. for all $m, i$ then $N_{j}=S_{j}$, $j=0,1, \ldots$. Therefore it suffices to study the prediction of $S_{j+\ell+1}$ given $N_{0}, \ldots, N_{j}$.

We will derive expressions for the predictors (2.1) and determine their errors. We start with the one-step ahead prediction problem, i.e., $\ell=0$.

2.1. One-step ahead prediction. We introduce some notation to be used throughout the paper. We will need the Laplace-Stieltjes transform of $M$, i.e.,

$$
L(\gamma)=E \mathrm{e}^{-\gamma M}=\sum_{m=0}^{\infty} q_{m} \mathrm{e}^{-\gamma m}, \quad \gamma \geq 0
$$

and its derivatives

$$
L^{(\ell)}(\gamma)=(-1)^{\ell} E\left(M^{\ell} \mathrm{e}^{-\gamma M}\right), \quad \gamma>0, \quad \ell=0,1, \ldots,
$$

with the convention that $L^{(0)}=L$. Moreover, define

$$
R_{\ell}(\gamma)=-\frac{L^{(\ell+1)}(\gamma)}{L^{(\ell)}(\gamma)}=\frac{E\left(M^{\ell+1} \mathrm{e}^{-\gamma M}\right)}{E\left(M^{\ell} \mathrm{e}^{-\gamma M}\right)}, \quad \gamma>0, \quad \ell=0,1, \ldots
$$

Finally, write

$$
\theta_{j}=\mu \sum_{d=0}^{j} p_{d}, \quad j=0,1, \ldots ;
$$

recall that $\mu$ is the Poisson rate of the number of payments per claim, and $\left(p_{k}\right)$ are the displacement probabilities in (1.4).

Next we formulate our main result on the prediction of $S_{j+1}$. Recall that the corresponding result for $N_{j+1}$ follows by setting $X_{m i}=1$ a.s. for all $m, i$.

Theorem 2.1. Assume that $E M<\infty$ and $E X_{11}<\infty$.

(1) The predictor $\widehat{S}_{j+1}$ of $S_{j+1}$ given $N_{0}, \ldots, N_{j}$ has the form

$$
\begin{aligned}
& (2.2) \quad E\left(S_{j+1} \mid N_{0}=n_{0}, \ldots, N_{j}=n_{j}\right)=\mu p_{j+1} E X_{11} R_{n_{0}+\cdots+n_{j}}\left(\theta_{j}\right), \\
& n_{0}, \ldots, n_{j}=0,1, \ldots, j=0,1, \ldots
\end{aligned}
$$

(2) Assume, in addition, that $\operatorname{var}(M)<\infty$ and $\operatorname{var}\left(X_{11}\right)<\infty$. Then the unconditional prediction error for $S_{j+1}, j=0,1, \ldots$, is given by

$$
\begin{aligned}
E\left(\left(S_{j+1}-\widehat{S}_{j+1}\right)^{2}\right) & =E\left(X_{11}^{2}\right) \mu p_{j+1} E M \\
& +\left(E X_{11} \mu p_{j+1}\right)^{2} E\left(M^{2}\right)-E\left(\widehat{S}_{j+1}^{2}\right) .
\end{aligned}
$$


(3) Assume, in addition, that $\operatorname{var}(M)<\infty$ and $\operatorname{var}\left(X_{11}\right)<\infty$. Then the conditional prediction error for $S_{j+1}$ given the past observations $N_{0}, \ldots, N_{j}$, $j=0,1, \ldots$, is

$$
\begin{aligned}
& \operatorname{var}\left(S_{j+1} \mid N_{0}=n_{0}, \ldots, N_{j}=n_{j}\right)=E\left(X_{11}^{2}\right) \mu p_{j+1} R_{n_{0}+\cdots+n_{j}}\left(\theta_{j}\right) \\
& +\left(E X_{11} \mu p_{j+1}\right)^{2} R_{n_{0}+\cdots+n_{j}}\left(\theta_{j}\right)\left[R_{n_{0}+\cdots+n_{j}+1}\left(\theta_{j}\right)-R_{n_{0}+\cdots+n_{j}}\left(\theta_{j}\right)\right] .
\end{aligned}
$$

Remark 2.2. Writing $\ell_{j}=n_{0}+\cdots+n_{j}, j=0,1, \ldots$, we observe by virtue of (2.2) that

$$
\begin{aligned}
& E\left(S_{j+1} \mid N_{0}=n_{0}, \ldots, N_{j}=n_{j}\right) \\
= & E\left(S_{j+1} \mid N_{0}=\ell_{0}, N_{0}+N_{1}=\ell_{1}, \ldots, N_{0}+\cdots+N_{j}=\ell_{j}\right) \\
= & E\left(S_{j+1} \mid N_{0}+\cdots+N_{j}=\ell_{j}\right),
\end{aligned}
$$

or, alternatively,

$$
\begin{aligned}
& E\left(S_{0}+\cdots+S_{j+1} \mid N_{0}+\cdots+N_{j}=\ell_{j}\right) \\
& =E X_{11} \ell_{j}+E\left(S_{j+1} \mid N_{0}+\cdots+N_{j}=\ell_{j}\right) .
\end{aligned}
$$

By virtue of (2.2), the conditional expectation (2.5) is in general not a linear function of $\ell_{j}$, in disagreement with Mack's procedure (1.2). In Section 4 we will give conditions on the distribution of $M$ ensuring that (2.5) is asymptotically linear as $\ell_{j} \rightarrow \infty$.

Remark 2.3. In Section 3 we will give a recursive algorithm for evaluating the quantities $L^{(\ell)}$ when the distribution of $M$ belongs to the $(a, b)$-class used for Panjer recursion.

Proof. (1) By the splitting property of the Poisson process, $\left(N_{j}\right)$ constitutes, conditionally on $M$, a sequence of independent Poisson random variables. Therefore

$$
E\left(N_{j+1} \mid N_{0}, \ldots, N_{j}, M\right)=M \mu p_{j+1}, \quad j=0,1, \ldots,
$$

and

$$
\widehat{S}_{j+1}=E X_{11} \mu p_{j+1} E\left(M \mid \mathcal{F}_{j}\right), \quad j=0,1, \ldots .
$$

Even more precisely, let $Z_{j l}$ denote the number of payments in the $l$ th payment stream, $l=1,2, \ldots$, which are executed in year $j=0,1, \ldots$ Then $\left(Z_{j l}\right)$ constitutes a double array of independent random variables with $Z_{j l} \sim$ $\operatorname{Poisson}\left(\mu p_{j}\right)$. Therefore for any $m, j=0,1, \ldots$ and $n_{0}, \ldots, n_{j}=0,1, \ldots$,

$$
\begin{aligned}
P & \left(N_{0}=n_{0}, \ldots, N_{j}=n_{j}, M=m\right) \\
& =q_{m} P\left(\sum_{l=1}^{m} Z_{0 l}=n_{0}\right) \cdots P\left(\sum_{l=1}^{m} Z_{j l}=n_{j}\right) \\
& =q_{m} \prod_{d=0}^{j} \mathrm{e}^{-m \mu p_{d}} \frac{\left(m \mu p_{d}\right)^{n_{d}}}{n_{d} !}
\end{aligned}
$$




$$
=q_{m} \mathrm{e}^{-m \theta_{j}} m^{\sum_{d=0}^{j} n_{d}} \prod_{d=0}^{j} \frac{\left(\mu p_{d}\right)^{n_{d}}}{n_{d} !} .
$$

We conclude that for $j \geq 0$,

$$
\begin{aligned}
P\left(M=m \mid N_{0}=n_{0}, \ldots, N_{j}=n_{j}\right)= & \frac{q_{m} \mathrm{e}^{-m \theta_{j}} m^{\sum_{d=0}^{j} n_{d}}}{\sum_{r=0}^{\infty} q_{r} \mathrm{e}^{-r} \theta_{j} r^{\sum_{d=0}^{j} n_{d}}}, \\
& m, n_{0}, \ldots, n_{j}=0,1, \ldots .
\end{aligned}
$$

In particular,

$$
\begin{aligned}
E\left(M \mid N_{0}=n_{0}, \ldots, N_{j}=n_{j}\right) & =\frac{\sum_{m=0}^{\infty} q_{m} \mathrm{e}^{-m \theta_{j}} m^{\sum_{d=0}^{j} n_{d}+1}}{\sum_{r=0}^{\infty} q_{r} \mathrm{e}^{-r \theta_{j}} r^{\sum_{d=0}^{j} n_{d}}} \\
& =R_{n_{0}+\cdots+n_{j}}\left(\theta_{j}\right) .
\end{aligned}
$$

We conclude, using (2.6), that (2.2) holds.

(2) \& (3) We start by calculating the prediction error of $S_{j+1}$ given the values $N_{0}, \ldots, N_{j}$. First observe that

$$
\operatorname{var}\left(S_{j+1} \mid N_{0}, \ldots, N_{j}, M\right)=M E\left(X_{11}^{2}\right) \mu p_{j+1} .
$$

Taking into account this relation and (2.1), we see that the conditional prediction error can be written as

$$
\begin{aligned}
& \operatorname{var}\left(S_{j+1} \mid \mathcal{F}_{j}\right)=E\left(X_{11}^{2}\right) E\left(N_{j+1} \mid \mathcal{F}_{j}\right) \\
& \quad+\left(E X_{11} \mu p_{j+1}\right)^{2} \operatorname{var}\left(M \mid \mathcal{F}_{j}\right) .
\end{aligned}
$$

Using (2.8), we can replace the conditional moments of $M$ by the corresponding derivatives of $L$, leading to (2.4). Taking expectations in (2.10), we obtain the prediction error

$$
\begin{aligned}
& E\left(\left(S_{j+1}-\widehat{S}_{j+1}\right)^{2}\right)=E\left[\operatorname{var}\left(S_{j+1} \mid \mathcal{F}_{j}\right)\right] \\
= & E\left(X_{11}^{2}\right) E N_{j+1}+\left(E X_{11} \mu p_{j+1}\right)^{2}\left[E\left(M^{2}\right)-E\left[\left(E\left(M \mid \mathcal{F}_{j}\right)\right)^{2}\right]\right] \\
= & E\left(X_{11}^{2}\right) \mu p_{j+1} E M+\left(E X_{11} \mu p_{j+1}\right)^{2} E\left(M^{2}\right)-E\left(\widehat{S}_{j+1}^{2}\right) .
\end{aligned}
$$

This finishes the proof.

Remark 2.4. A simple upper bound of the unconditional prediction error (2.3) is given by

$$
E\left[\left(S_{j+1}-\widehat{S}_{j+1}\right)^{2}\right] \leq E\left(X_{11}^{2}\right) \mu p_{j+1} E M+\left(E X_{11} \mu p_{j+1}\right)^{2} E\left(M^{2}\right) .
$$

Evaluation of $E\left(\widehat{S}_{l+1}^{2}\right)$ in (2.3) is complicated. Following the lines of the proof above, one can derive a more explicit expression for this term:

$$
\begin{aligned}
& E\left(\widehat{S}_{j+1}^{2}\right)=\left(E X_{11} \mu p_{j+1}\right)^{2} E\left[\left(E\left(M \mid \mathcal{F}_{j}\right)\right)^{2}\right] \\
= & \left(E X_{11} \mu p_{j+1}\right)^{2} E\left[\left(R_{N_{0}+\cdots+N_{j}}\left(\theta_{j}\right)\right)^{2}\right]
\end{aligned}
$$




$$
=\left(E X_{11} \mu p_{j+1}\right)^{2} \sum_{k=0}^{\infty} \sum_{m=0}^{\infty} P\left(N_{0}+\cdots+N_{j}=k, M=m\right)\left(R_{k}\left(\theta_{j}\right)\right)^{2} .
$$

Applying (2.7), the right-hand double sum turns into

$$
\begin{aligned}
& \sum_{k=0}^{\infty}\left[\sum_{m=0}^{\infty} q_{m} \mathrm{e}^{-m \theta_{j}} m^{k}\right]_{n_{0}+\cdots+n_{j}=k} \prod_{d=0}^{j} \frac{\left(\mu p_{d}\right)^{n_{d}}}{n_{d} !}\left(R_{k}\left(\theta_{j}\right)\right)^{2} \\
= & \mathrm{e}^{\theta_{j}} \sum_{k=0}^{\infty}(-1)^{k} \frac{\left(L^{(k+1)}\left(\theta_{j}\right)\right)^{2}}{L^{(k)}\left(\theta_{j}\right)} P\left(\Theta_{j}=k\right) \\
= & \mathrm{e}^{\theta_{j}} E\left((-1)_{j}^{\Theta} \frac{\left(L^{\left(\Theta_{j}+1\right)}\left(\theta_{j}\right)\right)^{2}}{L^{\left(\Theta_{j}\right)}\left(\theta_{j}\right)}\right),
\end{aligned}
$$

where $\Theta_{j}$ is $\operatorname{Poisson}\left(\theta_{j}\right)$ distributed.

2.2. Multi-step ahead prediction. In this subsection we consider the prediction problem for $\ell+1$ periods ahead. This means we are interested in the quantities $\widehat{S}_{j+\ell+1}, \ell=0,1, \ldots$, defined in (1.1), and the corresponding prediction errors.

Theorem 2.5. Assume that $E M<\infty$ and $E X_{11}<\infty$.

(1) The predictor $\widehat{S}_{j+\ell+1}$ of $S_{j+\ell+1}$ given $N_{0}, \ldots, N_{j}$ has the form

$$
\begin{aligned}
E\left(S_{j+\ell+1} \mid N_{0}=n_{0}, \ldots, N_{j}=n_{j}\right)= & E X_{11} \mu p_{j+\ell+1} R_{n_{0}+\cdots+n_{j}}\left(\theta_{j}\right), \\
& j, \ell=0,1, \ldots .
\end{aligned}
$$

(2) Assume, in addition, that $\operatorname{var}(M)<\infty$ and $\operatorname{var}\left(X_{11}\right)<\infty$. Then the unconditional prediction error for $S_{j+\ell+1}, j, \ell=0,1, \ldots$, is given by

$$
\begin{aligned}
E\left[\left(S_{j+\ell+1}-\widehat{S}_{j+\ell+1}\right)^{2}\right]= & E\left(X_{11}^{2}\right) \mu p_{j+\ell+1} E M \\
& +\left(E X_{11} \mu p_{j+\ell+1}\right)^{2} E\left(M^{2}\right)-E\left(\widehat{S}_{j+\ell+1}^{2}\right) .
\end{aligned}
$$

(3) Assume, in addition, that $\operatorname{var}(M)<\infty$ and $\operatorname{var}\left(X_{11}\right)<\infty$. Then the conditional prediction error for $S_{j+\ell+1}, j, \ell=0,1, \ldots$, is given by

$$
\begin{aligned}
& \operatorname{var}\left(S_{j+\ell+1} \mid N_{0}=n_{0}, \ldots, N_{j}=n_{j}\right) \\
&= E\left(X_{11}^{2}\right) \mu p_{j+\ell+1} R_{n_{0}+\cdots+n_{j}}\left(\theta_{j}\right) \\
&\left.\quad+\left(E X_{11} \mu p_{j+\ell+1}\right)^{2} R_{n_{0}+\cdots+n_{j}}\left(\theta_{j}\right)\left[R_{n_{0}+\cdots+n_{j}+1}\left(\theta_{j}\right)-R_{n_{0}+\cdots+n_{j}}\left(\theta_{j}\right)\right)\right] .
\end{aligned}
$$

Proof. (1) We start by observing that for $\ell \geq 0$,

$$
\widehat{S}_{j+\ell+1}=E\left[E\left(S_{j+\ell+1} \mid \mathcal{F}_{j+\ell}\right) \mid \mathcal{F}_{j}\right] .
$$

Hence, using (2.6), we obtain

$$
\widehat{S}_{j+\ell+1}=E X_{11} \mu p_{j+\ell+1} E\left[E\left(M \mid \mathcal{F}_{j+\ell}\right) \mid \mathcal{F}_{j}\right]
$$




$$
=E X_{11} \mu p_{j+\ell+1} E\left(M \mid \mathcal{F}_{j}\right) .
$$

Now use relation (2.9).

(2) \& (3) For the conditional prediction error for $S_{j+\ell+1}$, we observe that by $(2.10)$

$$
\begin{aligned}
& \operatorname{var}\left(S_{j+\ell+1} \mid \mathcal{F}_{j}\right) \\
= & E\left(\operatorname{var}\left(S_{j+\ell+1} \mid \mathcal{F}_{j+\ell}\right) \mid \mathcal{F}_{j}\right)+\operatorname{var}\left(E\left(S_{j+\ell+1} \mid \mathcal{F}_{j+\ell}\right) \mid \mathcal{F}_{j}\right) \\
= & E\left(X_{11}^{2}\right) \widehat{N}_{j+\ell+1}+\left(E X_{11} \mu p_{j+\ell+1}\right)^{2} E\left(\operatorname{var}\left(M \mid \mathcal{F}_{j+\ell}\right) \mid \mathcal{F}_{j}\right) \\
& +\left(E X_{11} \mu p_{j+\ell+1}\right)^{2} \operatorname{var}\left(E\left(M \mid \mathcal{F}_{j+\ell}\right) \mid \mathcal{F}_{j}\right) \\
= & E\left(X_{11}^{2}\right) \widehat{N}_{j+\ell+1}+\left(E X_{11} \mu p_{j+\ell+1}\right)^{2} \operatorname{var}\left(M \mid \mathcal{F}_{j}\right) .
\end{aligned}
$$

The conditional moments of $M$ can, once again, be expressed using (2.8). Taking expectations, we obtain the unconditional prediction error

$$
\begin{aligned}
& E\left[\left(S_{j+\ell+1}-\widehat{S}_{j+\ell+1}\right)^{2}\right]=E\left[\operatorname{var}\left(S_{j+\ell+1} \mid \mathcal{F}_{j}\right)\right] \\
& =E\left(X_{11}^{2}\right) \mu p_{j+\ell+1} E M+\left(E X_{11} \mu p_{j+\ell+1}\right)^{2}\left[E\left(M^{2}\right)-E\left(\left(E\left(M \mid \mathcal{F}_{j}\right)\right)^{2}\right)\right] \\
& =E\left(X_{11}^{2}\right) \mu p_{j+\ell+1} E M+\left(E X_{11} \mu p_{j+\ell+1}\right)^{2} E\left(M^{2}\right)-E\left(\widehat{S}_{j+\ell+1}^{2}\right) .
\end{aligned}
$$

Remark 2.6. Notice that

$$
\widehat{S}_{j+\ell+1}=\frac{p_{j+\ell+1}}{p_{j+1}} \widehat{S}_{j+1}, \quad \ell=0,1, \ldots,
$$

provided $p_{j+1}>0$. Moreover, if $p_{j+\ell+1}=0$ then $\widehat{S}_{j+\ell+1}=0$.

2.3. Conditionally independent payments. In this subsection we consider a slightly more general model. As before, we assume that the sequences $\left(X_{m k}\right)_{k=1,2, \ldots}, m=1,2, \ldots$, are iid and independent of the rest of random variables defining the model. We further assume that each sequence $\left(X_{m k}\right)_{k=1,2, \ldots}$ consists of conditionally iid random variables or, equivalently, that $\left(X_{m k}\right)_{k=1,2, \ldots}$ is exchangeable. This situation is similar to models in credibility theory, where the claim sizes occurring in an individual policy are assumed conditionally iid; see Mikosch [7], Chapters 5 and 6.

Since the random variables $\left(X_{m k}\right)_{m, k=1,2, \ldots}$ and $\left(Y_{m k}\right)_{m, k=1,2, \ldots}$ are independent the form of the one-step ahead predictor is again given by (2.1) but the prediction error changes.

Proposition 2.7. Assume $\operatorname{var}(M)<\infty$ and $\operatorname{var}\left(X_{11}\right)<\infty$. Then the unconditional prediction error for $S_{j+1}$ is given by

$$
\begin{aligned}
E\left(\left(S_{j+1}-\widehat{S}_{j+1}\right)^{2}\right)= & E M\left[E\left(X_{11}^{2}\right) \mu p_{j+1}+\operatorname{cov}\left(X_{11}, X_{12}\right)\left(\mu p_{j+1}\right)^{2}\right] \\
& +\left(E X_{11} \mu p_{j+1}\right)^{2} E\left(M^{2}\right)-E\left(\widehat{S}_{j+1}^{2}\right), \quad j=0,1, \ldots .
\end{aligned}
$$


The conditional prediction error of $S_{j+1}$ given $N_{0}, \ldots, N_{j}, j=0,1, \ldots$, has the form

$$
\begin{aligned}
& \operatorname{var}\left.S_{j+1} \mid N_{0}=n_{0}, \ldots, N_{j}=n_{j}\right) \\
&= {\left[E\left(X_{11}^{2}\right) \mu p_{j+1}+\operatorname{cov}\left(X_{11}, X_{12}\right)\left(\mu p_{j+1}\right)^{2}\right] R_{n_{0}+\cdots+n_{j}}\left(\theta_{j}\right) } \\
& \quad+\left(E X_{11} \mu p_{j+1}\right)^{2} R_{n_{0}+\cdots+n_{j}}\left(\theta_{j}\right)\left[R_{n_{0}+\cdots+n_{j}+1}\left(\theta_{j}\right)-R_{n_{0}+\cdots+n_{j}}\left(\theta_{j}\right)\right] .
\end{aligned}
$$

A comparison of this result with Theorem 2.1 shows that the prediction error increases by the additional term with the factor $\operatorname{cov}\left(X_{11}, X_{12}\right)$. It is non-negative as an application of the conditional Jensen inequality shows.

Proof. We start by calculating

$$
\begin{aligned}
& \operatorname{var}\left(S_{j+1} \mid N_{0}, \ldots, N_{j}, M\right)=M \operatorname{var}\left(\sum_{k=1}^{K_{1}} X_{1 k} I_{\left\{Y_{1 k}=j+1\right\}} \mid \mathcal{F}_{j}\right) \\
= & M E\left(\left(\sum_{k=1}^{K_{1}} X_{1 k} I_{\left\{Y_{1 k}=j+1\right\}}\right)^{2} \mid \mathcal{F}_{j}\right)-M\left(E X_{11} \mu p_{j+1}\right)^{2} .
\end{aligned}
$$

We observe that

$$
\begin{aligned}
& E\left(\left(\sum_{k=1}^{K_{1}} X_{1 k} I_{\left\{Y_{1 k}=j+1\right\}}\right)^{2} \mid \mathcal{F}_{j}\right)=E\left(\sum_{k=1}^{K_{1}} X_{1 k}^{2} I_{\left\{Y_{1 k}=j+1\right\}} \mid \mathcal{F}_{j}\right) \\
& +E\left(\sum_{k=1}^{K_{1}} \sum_{l=1, l \neq k}^{K_{1}} X_{1 k} X_{1 l} I_{\left\{Y_{1 k}=j+1\right\}} I_{\left\{Y_{1 l}=j+1\right\}} \mid \mathcal{F}_{j}\right) \\
= & {\left[E\left(X_{11}^{2}\right)-E\left(X_{11} X_{12}\right)\right] \mu p_{j+1}+E\left(X_{11} X_{12}\right) E\left(\left(\sum_{k=1}^{K_{1}} I_{\left\{Y_{1 k}=j+1\right\}}\right)^{2} \mid \mathcal{F}_{j}\right) } \\
= & E\left(X_{11}^{2}\right) \mu p_{j+1}+E\left(X_{11} X_{12}\right)\left(\mu p_{j+1}\right)^{2} .
\end{aligned}
$$

Here we used the fact that, by the exchangeability, $E\left(X_{11} X_{12}\right)=E\left(X_{1 k} X_{1 l}\right)$ for $k \neq l$. Overall, we obtain

$$
\operatorname{var}\left(S_{j+1} \mid N_{0}, \ldots, N_{j}, M\right)=M\left[E\left(X_{11}^{2}\right) \mu p_{j+1}+\operatorname{cov}\left(X_{11}, X_{12}\right)\left(\mu p_{j+1}\right)^{2}\right] .
$$

Therefore,

$$
\begin{aligned}
\operatorname{var}\left(S_{j+1} \mid \mathcal{F}_{j}\right)= & E\left(M \mid \mathcal{F}_{j}\right)\left(E\left(X_{11}^{2}\right) \mu p_{j+1}+\operatorname{cov}\left(X_{11}, X_{12}\right)\left(\mu p_{j+1}\right)^{2}\right) \\
& +\left(E X_{11} \mu p_{j+1}\right)^{2} \operatorname{var}\left(M \mid \mathcal{F}_{j}\right) .
\end{aligned}
$$

For the unconditional prediction error we have

$$
\begin{aligned}
& E\left[\left(S_{j+1}-\widehat{S}_{j+1}\right)^{2}\right]=E M\left[E\left(X_{11}^{2}\right) \mu p_{j+1}+\operatorname{cov}\left(X_{11}, X_{12}\right)\left(\mu p_{j+1}\right)^{2}\right] \\
& +E\left(M^{2}\right)\left(E X_{11} \mu p_{j+1}\right)^{2}-\left(E X_{11} \mu p_{j+1}\right)^{2} E\left[\left(E\left(M \mid \mathcal{F}_{j}\right)\right)^{2}\right] \\
= & E M\left[E\left(X_{11}^{2}\right) \mu p_{j+1}+\operatorname{cov}\left(X_{11}, X_{12}\right)\left(\mu p_{j+1}\right)^{2}\right]
\end{aligned}
$$




$$
+E\left(M^{2}\right)\left(E X_{11} \mu p_{j+1}\right)^{2}-E\left(\widehat{S}_{j+1}^{2}\right) \text {. }
$$

\section{Prediction in the $(a, b)$-Class}

In the previous sections we have learned that, for predicting the values $N_{j+\ell+1}$ and $S_{j+\ell+1}, \ell \geq 0$, given $N_{0}, \ldots, N_{j}$, it is crucial to be able to evaluate the derivatives $(-1)^{l} L^{(l)}(\gamma)=E\left(M^{l} \mathrm{e}^{-\gamma M}\right)$. In this section we assume that the distribution of $M$ belongs to the $(a, b)$-class which is used in the Panjer recursive algorithm; see Mikosch [7], Section 3.3. This class is given by the recursive relation

$$
q_{0}>0, \quad q_{m}=(a+b / m) q_{m-1}, \quad m=1,2, \ldots, \quad a, b \in \mathbb{R} .
$$

This class contains exactly three non-degenerate distributions.

(1) The Poisson( $b$ ) distribution with $a=0, b>0$.

(2) The $\operatorname{Bin}(n, p)$ distribution with $a<0, a=-p /(1-p), b=-a(n+1)$ and $p \in(0,1), n \geq 1$.

(3) The negative binomial distribution with parameter $(p, v)$ :

$$
q_{m}=\left(\begin{array}{c}
v+m-1 \\
m
\end{array}\right) p^{v}(1-p)^{m}, \quad m=0,1, \ldots, \quad p \in(0,1), \quad v>0 .
$$

$$
\text { where } 0<a=1-p, b=(1-p)(v-1) \text {. }
$$

We will derive a recursion for the expressions $(-1)^{l} L^{(l)}(\gamma)=E\left(M^{l} \mathrm{e}^{-\gamma M}\right)$. Using the $(a, b)$-structure and the binomial formula, we have for $l \geq 1$,

$$
\begin{aligned}
& (-1)^{l} L^{(l)}(\gamma)=\sum_{m=1}^{\infty} \mathrm{e}^{-\gamma m} m^{l}(a+b / m) q_{m-1} \\
= & a \mathrm{e}^{-\gamma} \sum_{m=0}^{\infty} \mathrm{e}^{-\gamma m}(m+1)^{l} q_{m}+b \mathrm{e}^{-\gamma} \sum_{m=0}^{\infty} \mathrm{e}^{-\gamma m}(m+1)^{l-1} q_{m} \\
= & a \mathrm{e}^{-\gamma} \sum_{m=0}^{\infty} \mathrm{e}^{-\gamma m} \sum_{r=0}^{l}\left(\begin{array}{l}
l \\
r
\end{array}\right) m^{r} q_{m}+b \mathrm{e}^{-\gamma} \sum_{m=0}^{\infty} \mathrm{e}^{-\gamma m} \sum_{r=0}^{l-1}\left(\begin{array}{c}
l-1 \\
r
\end{array}\right) m^{r} q_{m} \\
= & a \mathrm{e}^{-\gamma}(-1)^{l} L^{(l)}(\gamma)+\mathrm{e}^{-\gamma} \sum_{r=0}^{l-1}\left[a\left(\begin{array}{c}
l \\
r
\end{array}\right)+b\left(\begin{array}{c}
l-1 \\
r
\end{array}\right)\right](-1)^{r} L^{(r)}(\gamma) .
\end{aligned}
$$

Hence

$$
(-1)^{l} L^{(l)}(\gamma)=\frac{\mathrm{e}^{-\gamma}}{1-a \mathrm{e}^{-\gamma}} \sum_{r=0}^{l-1}\left[a\left(\begin{array}{l}
l \\
r
\end{array}\right)+b\left(\begin{array}{c}
l-1 \\
r
\end{array}\right)\right](-1)^{r} L^{(r)}(\gamma) .
$$

Notice that these formulas are meaningful because $a$ is always smaller than

1. Now we consider the three different classes of $(a, b)$-distributions. 
Proposition 3.1. Assume that the distribution of $M$ is in the $(a, b)$-class. (1) In the Poisson(b) case, $a=0, b>0$, we have

$$
\begin{aligned}
L(\gamma) & =\mathrm{e}^{-b\left(1-\mathrm{e}^{-\gamma}\right)}, \quad \gamma \geq 0 \\
(-1)^{l} L^{(l)}(\gamma) & =\mathrm{e}^{-\gamma} b \sum_{r=0}^{l-1}\left(\begin{array}{c}
l-1 \\
r
\end{array}\right)(-1)^{r} L^{(r)}(\gamma), \quad l \geq 1 .
\end{aligned}
$$

(2) In the $\operatorname{Bin}(n, p)$ case, $a=-p /(1-p), b=p(n+1) /(1-p), n=1,2, \ldots$ and $p \in(0,1)$, we have for $l \geq 1$,

$$
\begin{aligned}
L(\gamma) & =\left(1-p\left(1-\mathrm{e}^{-\gamma}\right)\right)^{n}, \quad \gamma \geq 0, \\
(-1)^{l} L^{(l)}(\gamma) & =\frac{p \mathrm{e}^{-\gamma}}{1-p\left(1-\mathrm{e}^{-\gamma}\right)} \sum_{r=0}^{l-1}\left(\begin{array}{l}
l \\
r
\end{array}\right)\left[(n+1) \frac{l-r}{l}-1\right](-1)^{r} L^{(r)}(\gamma) .
\end{aligned}
$$

(3) In the negative binomial case, $a=1-p, b=(1-p)(v-1), p \in(0,1)$ and $v>0$, we have for $l \geq 1$,

$$
\begin{aligned}
L(\gamma) & =\left(\frac{p}{1-(1-p) \mathrm{e}^{-\gamma}}\right)^{v}, \quad \gamma \geq 0, \\
(-1)^{l} L^{(l)}(\gamma) & =\frac{(1-p) \mathrm{e}^{-\gamma}}{1-(1-p) \mathrm{e}^{-\gamma}} \sum_{r=0}^{l-1}\left(\begin{array}{l}
l \\
r
\end{array}\right)\left[(v-1) \frac{l-r}{l}+1\right](-1)^{r} L^{(r)}(\gamma) .
\end{aligned}
$$

Remark 3.2. In the Poisson case, one can also get a different recursion for $L^{(k)}$. Introduce the polynomial $H_{n}$ of degree $n$ by the recursion

$$
H_{0}(x)=1, \quad H_{n}(x)=-x\left[H_{n-1}^{\prime}(x)+H_{n-1}(x)\right], \quad n \geq 1, \quad x>0 .
$$

Then calculation yields

$$
L^{(k)}(\gamma)=H_{k}\left(b \mathrm{e}^{-\gamma}\right) L(\gamma), \quad k \geq 0, \quad \gamma>0 .
$$

In particular, $R_{k}(\gamma)$ is a rational function of $b \mathrm{e}^{-\gamma}$ for each $k \geq 0$ :

$$
R_{k}(\gamma)=-\frac{H_{k+1}\left(b \mathrm{e}^{-\gamma}\right)}{H_{k}\left(b \mathrm{e}^{-\gamma}\right)}=b \mathrm{e}^{-\gamma}\left(1+\frac{H_{k}^{\prime}\left(b \mathrm{e}^{-\gamma}\right)}{H_{k}\left(b \mathrm{e}^{-\gamma}\right)}\right) .
$$

Remark 3.3. Extensions of the $(a, b)$-class were considered in Hess et al. [1]. They introduced distributions $\left(q_{m}\right)_{m=0,1, \ldots}$ satisfying the $(a, b)$-condition (3.1) with $q_{0}, \ldots, q_{k}=0$ for some $k \geq 0$ and $q_{k+1}>0$. The calculations leading to the recursion (3.2) for $L^{(l)}$ remain valid in this case as well.

Remark 3.4. The quantities $(-1)^{l} L^{(l)}(\gamma)$ grow rapidly as a function of $l$ and therefore standard software delivers the value $\infty$ even for moderately large values $l$. This numerical problem can be avoided by writing (3.2) in terms of the ratios $R_{r}(\gamma)$ which are relevant for the prediction formulae considered in the previous sections:

$$
R_{l}(\gamma)=\frac{\mathrm{e}^{-\gamma}}{1-a \mathrm{e}^{-\gamma}} \sum_{r=0}^{l}\left[a\left(\begin{array}{c}
l+1 \\
r
\end{array}\right)+b\left(\begin{array}{l}
l \\
r
\end{array}\right)\right]\left(R_{r}(\gamma) \cdots R_{l-1}(\gamma)\right)^{-1}
$$


The latter recursion for $R_{l}$ avoids the direct calculation of the large quantities $\left|L^{(l)}(\gamma)\right|$

\section{The asymptotic Behavior of the PREDiCtion}

4.1. The behavior of $R_{k}(\gamma)$ as $k \rightarrow \infty$. In this subsection we study the asymptotic behavior of the predictors $E\left(S_{j+1} \mid N_{0}=n_{0}, \ldots, N_{j}=n_{j}\right)$, $j=0,1, \ldots$, when the number of payments $N_{0}+\cdots+N_{j}=n_{0}+\cdots+n_{j}=$ $k \rightarrow \infty$. The same discussion will apply equally to the multi-step predictors $E\left(S_{j+\ell+1} \mid N_{0}=n_{0}, \ldots, N_{j}=n_{j}\right), j, \ell=0,1, \ldots$ In view of the results in Theorem 2.1 one needs to study the asymptotic behavior of the ratios $R_{k}(\gamma)$ as $k \rightarrow \infty$.

The interest in the asymptotic behavior of $R_{k}(\gamma)$ as $k \rightarrow \infty$ is triggered, in particular, by a comparison with Mack's procedure (1.2). The latter declares the predictor of $N_{j+\ell+1}$ given $N_{0}, \ldots, N_{j}$ to be a linear function of $k=N_{0}+\cdots+N_{j}$. In our setting, this predictor is a multiple of $R_{k}(\gamma)$ which has no reason to be linear. However, this observation does not exclude the case that the limit $k^{-1} R_{k}(\gamma)$ exists, is finite and positive. In such cases $R_{k}(\gamma)$ would be approximately linear for large $k$, as in Mack's procedure.

The following result yields a sufficient condition for asymptotic linearity of $R_{k}(\gamma)$.

Lemma 4.1. Assume that $q_{m}>0$ for $m \geq m_{0}$ and the limit

$$
\lim _{m \rightarrow \infty} \frac{q_{m}}{q_{m-1}}=\mathrm{e}^{-\tau} \in(0,1]
$$

exists. Then

$$
\lim _{k \rightarrow \infty} \frac{R_{k}(\gamma)}{k}=\frac{1}{\gamma+\tau}
$$

Proof. Let $\epsilon \in(0,1)$. We decompose $(-1)^{k} L^{(k)}(\gamma)$ for fixed $\gamma$ :

$$
\begin{aligned}
(-1)^{k} L^{(k)}(\gamma) & =\left(\sum_{m<\frac{k(1-\epsilon)}{\gamma+\tau}}+\sum_{m \in\left[\frac{k(1-\epsilon)}{\gamma+\tau}, \frac{k(1+\epsilon)}{\gamma+\tau}\right]}+\sum_{m>\frac{k(1+\epsilon)}{\gamma+\tau}}\right) m^{k} q_{m} \mathrm{e}^{-\gamma m} \\
& =I_{1}(\epsilon)+I_{2}(\epsilon)+I_{3}(\epsilon) .
\end{aligned}
$$

We start by studying $I_{3}(\epsilon)$. For $m>k(1+\epsilon) /(\gamma+\tau)$,

$$
\begin{gathered}
\frac{(m+1)^{k} \mathrm{e}^{-(m+1) \gamma}}{m^{k} \mathrm{e}^{-m \gamma}}=\mathrm{e}^{-\gamma}\left(1+m^{-1}\right)^{k} \\
\leq \mathrm{e}^{-\gamma}\left(\left(1+m^{-1}\right)^{m}\right)^{\frac{\gamma+\tau}{1+\epsilon}} \leq \mathrm{e}^{-\gamma+(\gamma+\tau) /(1+\epsilon)} .
\end{gathered}
$$

Choose $\delta \in(0, \epsilon /(1+\epsilon))$. For $k$ large enough, which implies that $m>$ $k(1+\epsilon) /(\gamma+\tau)$ is large enough, we have in view of (4.1),

$$
\frac{q_{m+1}}{q_{m}} \leq \mathrm{e}^{-\tau(1-\delta)}
$$


Combining these two bounds, we obtain

$$
\frac{q_{m+1}(m+1)^{k} \mathrm{e}^{-(m+1) \gamma}}{q_{m} m^{k} \mathrm{e}^{-m \gamma}} \leq \mathrm{e}^{-\gamma+(\gamma+\tau) /(1+\epsilon)} \mathrm{e}^{-\tau(1-\delta)}=: f(\epsilon, \delta)<1,
$$

where we used the fact that $\delta \in(0, \epsilon /(1+\epsilon))$. Therefore for large $k$,

$$
I_{3}(\epsilon) \leq \frac{1}{1-f(\epsilon, \delta)} q_{\lceil k(1+\epsilon) /(\gamma+\tau)\rceil}\left\lceil\frac{k(1+\epsilon)}{\gamma+\tau}\right\rceil^{k} \mathrm{e}^{-\lceil k(1+\epsilon) /(\gamma+\tau)\rceil \gamma} .
$$

Further, for large $k$ the index set in $I_{2}(\epsilon)$ contains the point $[k /(\gamma+\tau)]$. Therefore we obtain a lower bound, valid for large $k$ :

$$
I_{2}(\epsilon) \geq q_{[k /(\gamma+\tau)]}\left[\frac{k}{\gamma+\tau}\right]^{k} \mathrm{e}^{-\gamma[k /(\gamma+\tau)]} .
$$

A combination of (4.3) aud (4.4) yields

$$
\begin{gathered}
\frac{I_{3}(\epsilon)}{I_{2}(\epsilon)} \leq \frac{1}{1-f(\epsilon, \delta)} \frac{q_{\lceil k(1+\epsilon) /(\gamma+\tau)\rceil}}{q_{[k /(\gamma+\tau)]}}\left(\frac{\lceil k(1+\epsilon) /(\gamma+\tau)\rceil}{[k /(\gamma+\tau)]}\right)^{k} \\
\mathrm{e}^{-[\lceil k(1+\epsilon) /(\gamma+\tau)\rceil-[k /(\gamma+\tau)]] \gamma} .
\end{gathered}
$$

By virtue of (4.1), for small $\alpha \in(0,1)$ and large $k$,

$$
\begin{aligned}
\frac{q_{\lceil k(1+\epsilon) /(\gamma+\tau)]}}{q_{[k /(\gamma+\tau)]}} & \leq \mathrm{e}^{-\tau(1-\alpha)(\lceil k(1+\epsilon) /(\gamma+\tau)\rceil-[k /(\gamma+\tau)])} \\
& \leq \mathrm{e}^{-(\tau /(\gamma+\tau)) k(1-\alpha) \epsilon} .
\end{aligned}
$$

Furthermore, for large $k$ and some positive constant $c_{1}$,

$$
\begin{aligned}
& \left(\frac{\lceil k(1+\epsilon) /(\gamma+\tau)\rceil}{[k /(\gamma+\tau)]}\right)^{k} \leq\left(\frac{k(1+\epsilon) /(\gamma+\tau)+1}{k /(\gamma+\tau)-1}\right)^{k} \\
= & (1+\epsilon)^{k}\left(1+\frac{1}{k(1+\epsilon) /(\gamma+\tau)}\right)^{k}\left(1-\frac{1}{k /(\gamma+\tau)}\right)^{-k} \\
\leq & c_{1}(1+\epsilon)^{k} .
\end{aligned}
$$

Finally,

$$
\mathrm{e}^{-[\lceil k(1+\epsilon) /(\gamma+\tau)]-[k /(\gamma+\tau)]] \gamma} \leq \mathrm{e}^{-\gamma k \epsilon /(\gamma+\tau)} .
$$

Collecting the above bounds and choosing $\alpha$ such that

$$
\mathrm{e}^{\epsilon(\tau(1-\alpha)+\gamma) /(\gamma+\tau)}>(1+\alpha)(1+\epsilon),
$$

we obtain, for some positive constant $c_{2}$,

$$
\frac{I_{3}(\epsilon)}{I_{2}(\epsilon)} \leq c_{2}(1+\epsilon)^{k} \mathrm{e}^{-k \epsilon(\tau(1-\alpha)+\gamma) /(\gamma+\tau)} \leq c_{2}\left(\frac{1}{1+\alpha}\right)^{k} .
$$

Hence $I_{3}(\epsilon)=o\left(I_{2}(\epsilon)\right)$ as $k \rightarrow \infty$. 
Next we turn to the estimation of $I_{1}(\epsilon)$. Once again, let $\delta \in(0,1)$ be small and choose $m_{0}$ so large that

$$
\frac{q_{m+1}}{q_{m}} \geq \mathrm{e}^{-\tau(1+\delta)} \quad\left(1+\frac{1}{m}\right)^{m} \geq \mathrm{e}^{1-\delta}, \quad m \geq m_{0} .
$$

We further decompose $I_{1}(\epsilon)$ :

$$
I_{1}(\epsilon)=\left(\sum_{m<m_{0}}+\sum_{m \in\left[m_{0}, k \frac{1-\epsilon}{\gamma+\tau}\right)}\right) m^{k} q_{m} \mathrm{e}^{-\gamma m}=I_{11}(\epsilon)+I_{12}(\epsilon) .
$$

Trivially, $I_{11}(\epsilon)=o\left(I_{2}(\epsilon)\right)$ as $k \rightarrow \infty$. For $m \in[k, k(1-\epsilon) /(\gamma+\tau))$ we have by $(4.5)$

$$
\left(1+m^{-1}\right)^{k} \geq \mathrm{e}^{(k / m)(1-\delta)} \geq \mathrm{e}^{(\gamma+\tau)(1-\delta) /(1-\epsilon)},
$$

and, therefore,

$\frac{q_{m+1}(m+1)^{k} \mathrm{e}^{-(m+1) \gamma}}{q_{m} m^{k} \mathrm{e}^{-m \gamma}} \geq \mathrm{e}^{-\gamma} \mathrm{e}^{-\tau(1+\delta)} \mathrm{e}^{-(\gamma+\tau)(1-\delta) /(1-\epsilon)}=g(\epsilon, \delta)>1$,

if we choose $\delta \in(0,1)$ so small that $(1-\delta) /(1-\epsilon)<1+\delta$. Therefore for $k$ large,

$$
I_{12}(\epsilon) \leq \frac{1}{1-(g(\epsilon, \delta))^{-1}} q_{\lfloor k(1-\epsilon) /(\gamma+\tau)\rfloor}\left\lfloor\frac{k(1-\epsilon)}{\gamma+\tau}\right\rfloor^{k} \mathrm{e}^{-\lfloor k(1-\epsilon) /(\gamma+\tau)\rfloor \gamma},
$$

and an argument similar to the one above implies that $I_{12}(\epsilon)=o\left(I_{2}(\epsilon)\right)$ as $k \rightarrow \infty$ for every $\epsilon \in(0,1)$.

We conclude that for $\epsilon \in(0,1)$, as $k \rightarrow \infty$

$$
R_{k}(\gamma) \sim \frac{\sum_{m \in\left[\frac{k(1-\epsilon)}{\gamma+\tau}, \frac{k(1+\epsilon)}{\gamma+\tau}\right]} m^{k+1} q_{m} \mathrm{e}^{-\gamma m}}{\sum_{m \in\left[\frac{k(1-\epsilon)}{\gamma+\tau}, \frac{k(1+\epsilon)}{\gamma+\tau}\right]} m^{k} q_{m} \mathrm{e}^{-\gamma m}} \in\left[\frac{k(1-\epsilon)}{\gamma+\tau}, \frac{k(1+\epsilon))}{\gamma+\tau}\right],
$$

and, hence, relation (4.2) is immediate. This concludes the proof.

In view of Lemma 4.1 and Theorem 2.1 we conclude that, under suitable conditions on the distribution of $M$, the predictor for $S_{j+1}$ given $N_{0}, \ldots, N_{j}$ is asymptotically linear.

Corollary 4.2. Assume that $E X_{11}<\infty$ and that the distribution of $M$ satisfies condition (4.1). Then, as $n_{0}+\cdots+n_{j} \rightarrow \infty$,

$$
E\left(S_{j+1} \mid N_{0}=n_{0}, \ldots, N_{j}=n_{j}\right) \sim \frac{E X_{11} \mu p_{j+1}}{\tau+\theta_{j}}\left(n_{0}+\cdots+n_{j}\right) .
$$

In the rest of this subsection we study the behavior of $R_{k}(\gamma)$ for large $k$ for the distributions in the $(a, b)$-class introduced in Section 3.

4.1.1. The negative binomial distribution. The negative binomial distribution is the only member of the $(a, b)$-class satisfying the condition (4.1) with $\mathrm{e}^{-\tau}=1-p$. Hence Corollary 4.2 applies. The asymptotically linear behavior of $R_{k}(\gamma)$ is nicely illustrated in the right graph of Figure 4.3. 

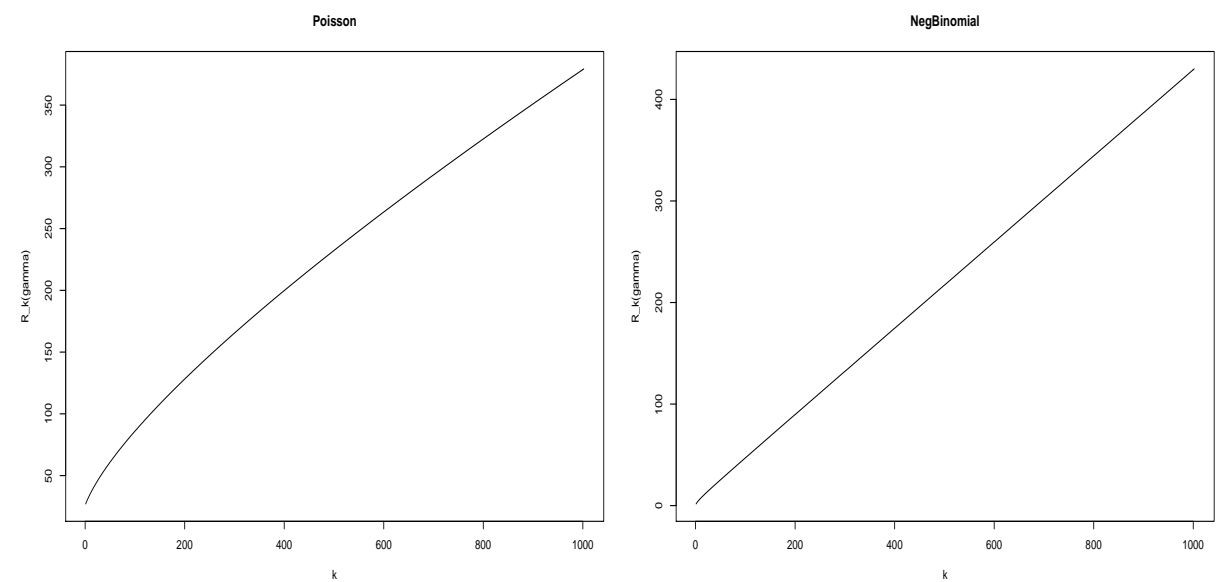

Figure 4.3. The ratio $R_{k}(2)$ for Poisson(200) distributed $M$ (left) and for negative binomial $M$ with parameters $p=0.1, v=12.1$ (right).

4.1.2. The binomial distribution. In this case, it is clear that, as $k \rightarrow \infty$,

$$
R_{k}(\gamma)=\frac{\sum_{m=1}^{n}(m / n)^{k} m \mathrm{e}^{-\gamma m} q_{m}}{\sum_{m=1}^{n}(m / n)^{k} \mathrm{e}^{-\gamma m} q_{m}} \rightarrow n .
$$

The same result holds for any distribution $\left(q_{m}\right)_{m=0, \ldots, n}, n \geq 1$, with $q_{n}>0$. In this case, if $E X_{11}<\infty$, then, as $n_{0}+\cdots+n_{j} \rightarrow \infty$,

$$
E\left(S_{j+1} \mid N_{0}=n_{0}, \ldots, N_{j}=n_{j}\right) \rightarrow n E X_{11} \mu p_{j+1} .
$$

4.1.3. The Poisson distribution. If $M$ is Poisson(b) distributed, then

$$
E\left(M^{k} \mathrm{e}^{-\gamma M}\right)=\mathrm{e}^{-b\left(1-\mathrm{e}^{-\gamma}\right)} \sum_{m=1}^{\infty} m^{k}\left(b / \mathrm{e}^{\gamma}\right)^{m} \frac{1}{m !} \mathrm{e}^{-b \mathrm{e}^{-\gamma}}, \quad k \geq 1 .
$$

Hence, the ratio $R_{k}(\gamma)$ is equal to the ratio of the moments $E\left(M^{\prime}\right)^{k+1} / E\left(M^{\prime}\right)^{k}$ for some Poisson random variable $M^{\prime}$ with a different mean, say, $\lambda$. In the sequel we study, therefore, the asymptotic behavior, as $k \rightarrow \infty$, of such ratios. For simplicity, we use the notation $E M^{k+1} / E M^{k}$ instead of the proper $E\left(M^{\prime}\right)^{k+1} / E\left(M^{\prime}\right)^{k}$.

The proof of the following lemma is similar to the proof of Lemma 4.1. We sketch the argument.

Lemma 4.4. Let $M$ be Poisson $(\lambda)$ distributed. Then, as $k \rightarrow \infty$,

$$
\frac{E M^{k+1}}{E M^{k}} \sim \frac{k}{\log k} .
$$

Proof. For $\epsilon \in(0,1)$ we decompose $E M^{k}$ as

$$
E M^{k}=\left(\sum_{m<\frac{k(1-\epsilon)}{\log k}}+\sum_{m \in\left[\frac{k(1-\epsilon)}{\log k}, \frac{k(1+\epsilon)}{\log k}\right]}+\sum_{m>\frac{k(1+\epsilon)}{\log k}}\right) m^{k} \mathrm{e}^{-\lambda} \frac{\lambda^{m}}{m !}
$$




$$
=I_{1}(\epsilon)+I_{2}(\epsilon)+I_{3}(\epsilon) \text {. }
$$

Beginning with $I_{3}(\epsilon)$, it is straightforward to check, using Stirling's formula, that

$$
\sup _{m>\frac{k(1+\epsilon)}{\log k}} \frac{(m+1)^{k} \lambda^{m+1} /(m+1) !}{m^{k} \lambda^{m} / m !} \leq \frac{\lambda \log k}{1+\epsilon} k^{-\epsilon /(1+\epsilon)} \rightarrow 0
$$

as $k \rightarrow \infty$. Hence, as $k \rightarrow \infty$,

$$
I_{3}(\epsilon)=(1+o(1))\left(m_{*}^{+, \epsilon}\right)^{k} \mathrm{e}^{-\lambda} \frac{\lambda^{m_{*}^{+, \epsilon}}}{\left(m_{*}^{+, \epsilon}\right) !},
$$

where

$$
m_{*}=\lfloor k / \log k\rfloor, m_{*}^{+, \epsilon}=\left\lceil(1+\epsilon) m_{*}\right\rceil .
$$

It is clear that for large $k$,

$$
I_{2}(\epsilon) \geq m_{*}^{k} \mathrm{e}^{-\lambda} \frac{\lambda^{m_{*}}}{m_{*} !} .
$$

Therefore, as $k \rightarrow \infty$,

$$
\frac{I_{3}(\epsilon)}{I_{2}(\epsilon)}=\mathrm{e}^{k(\log (1+\epsilon)-\epsilon+o(1))} .
$$

Since $\log (1+\epsilon)<\epsilon$, we conclude that for every $\epsilon>0$,

$$
\frac{I_{3}(\epsilon)}{I_{2}(\epsilon)} \rightarrow 0 \text { as } k \rightarrow \infty
$$

Similarly, as $k \rightarrow \infty$,

$$
I_{1}(\epsilon)=(1+o(1))\left(m_{*}^{-, \epsilon}\right)^{k} \mathrm{e}^{-\lambda} \frac{\lambda^{m_{*}^{-, \epsilon}}}{\left(m_{*}^{-, \epsilon}\right) !},
$$

where

$$
m_{*}^{-, \epsilon}=\left\lfloor(1-\epsilon) m_{*}\right\rfloor,
$$

from which it is easy to check that, as $k \rightarrow \infty$,

$$
\frac{I_{1}(\epsilon)}{I_{2}(\epsilon)}=\mathrm{e}^{k(\log (1-\epsilon)+\epsilon+o(1))} .
$$

Since $-\log (1-\epsilon)>\epsilon$ for $0<\epsilon<1$, we conclude that for every $\epsilon>0$,

$$
\frac{I_{1}(\epsilon)}{I_{2}(\epsilon)} \rightarrow 0 \quad \text { as } k \rightarrow \infty \text {. }
$$

That is, for every $\epsilon>0$,

$$
E M^{k}=(1+o(1)) \sum_{m \in\left[\frac{k(1-\epsilon)}{\log k}, \frac{k(1+\epsilon)}{\log k}\right]} m^{k} \mathrm{e}^{-\lambda} \frac{\lambda^{m}}{m !}
$$


as $k \rightarrow \infty$ and, hence,

$$
\begin{aligned}
\frac{E M^{k+1}}{E M^{k}}= & (1+o(1)) \frac{\sum_{m \in\left[\frac{k(1-\epsilon)}{\log k}, \frac{k(1+\epsilon)}{\log k}\right]} m^{k+1} \mathrm{e}^{-\lambda} \frac{\lambda^{m}}{m !}}{\sum_{m \in\left[\frac{k(1-\epsilon)}{\log k}, \frac{k(1+\epsilon)}{\log k}\right]} m^{k} \mathrm{e}^{-\lambda} \frac{\lambda^{m}}{m !}} \\
& \in\left[(1+o(1)) \frac{k(1-\epsilon)}{\log k},(1+o(1)) \frac{k(1+\epsilon)}{\log k}\right] .
\end{aligned}
$$

Hence the statement of the lemma.

Taking into account Lemma 4.4 and the remark preceeding it, we conclude that, if $M$ is Poisson(b) distributed, then, as $n_{0}+\cdots+n_{j} \rightarrow \infty$,

$$
E\left(S_{j+1} \mid N_{0}=n_{0}, \ldots, N_{j}=n_{j}\right) \sim E X_{11} \mu p_{j+1} \frac{n_{0}+\cdots+n_{j}}{\log \left(n_{0}+\cdots+n_{j}\right)} .
$$

The asymptotic behavior of $R_{k}(\gamma)$ prescribed by (4.6) is nicely illustrated in the left graph of Figure 4.3.

4.2. The behavior of $R_{k}(\gamma)$ for large $\gamma$. When evaluating the ratio $R_{k}(\gamma)$ numerically, one observes a rather unusual phenomenon for large values of $\gamma: R_{k}(\gamma)$ oscillates rather strongly for moderately large values of $k$, whereas this effect gradually disappears when $k$ becomes even larger. For small values of $\gamma$ this behavior cannot be observed. A computer graph of $R_{k}(\gamma)$ which exhibits this staircase-like behavior is illustrated in Figure 4.5. Below we give a limit theorem explaining this phenomenon.

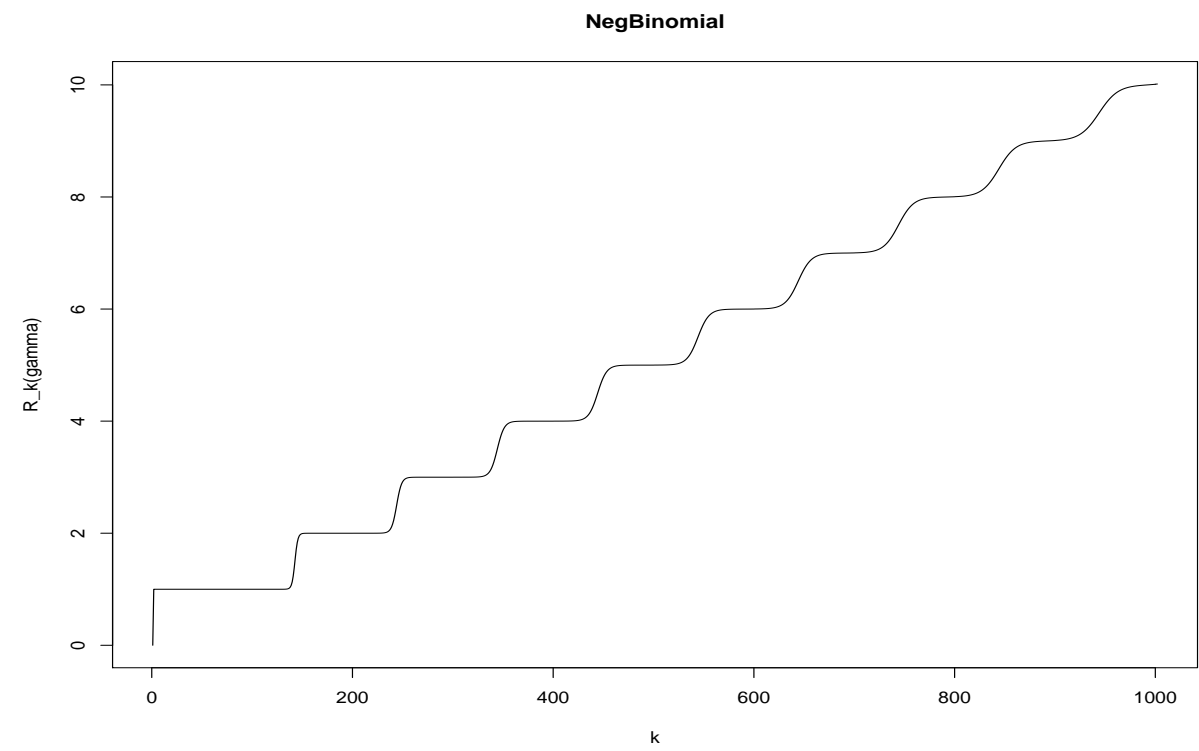

Figure 4.5. The ratio $R_{k}(100)$ for negative binomial $M$ with parameters $p=0.1$ and $v=12.1$. 
Assume that

$$
\gamma \rightarrow \infty, \quad k=k(\gamma) \quad \text { and } \quad k / \gamma \rightarrow t \in(0, \infty) .
$$

For $t>0$ we consider the strictly convex function

$$
h_{t}(s)=s-t \log s, \quad s>0,
$$

which reaches its minimum at $s=t$. Therefore the sequence $\left(h_{t}(m)\right)_{m=1,2, \ldots}$ reaches its minimum either at $m=m_{-}(t)=\lfloor t\rfloor$ if $m_{-}(t) \geq 1$ or at $m=$ $m_{+}(t)=\lceil t\rceil$, and it is possible that the values of $h_{t}$ at $m_{-}(t)$ and $m_{+}(t)$ coincide. Let us assume that there exists a unique value $m=m_{*}(t)$ at which $h_{t}$ is minimized. We have for $k \geq 1$,

$$
E\left(M^{k} \mathrm{e}^{-\gamma M}\right)=q_{m_{*}(t)}\left(m_{*}(t)\right)^{k} \mathrm{e}^{-\gamma m_{*}(t)}+\sum_{m \neq m_{*}(t)} m^{k} \mathrm{e}^{-\gamma m} q_{m} .
$$

By virtue of (4.7),

$$
\begin{aligned}
q_{m_{*}(t)}\left(m_{*}(t)\right)^{k} \mathrm{e}^{-\gamma m_{*}(t)} & =q_{m_{*}(t)} \mathrm{e}^{-\gamma h_{k / \gamma}\left(m_{*}(t)\right)} \\
& \rightarrow q_{m_{*}(t)} \mathrm{e}^{-\gamma h_{t}\left(m_{*}(t)\right)}
\end{aligned}
$$

The assumption that the minimum of $h_{t}(m)$ is unique for a given $t$ implies that the minimum of $h_{u}(m)$ is also unique for $u$ in a small neighborhood of $t$ and achieved at $m_{*}(t)$. Hence, for $u$ sufficiently close to $t$,

$$
\begin{aligned}
\min _{m \neq m_{*}(t)} h_{u}(m) & =\min \left(h_{u}\left(m_{*}(t)-1\right), h_{u}\left(m_{*}(t)+1\right)\right) \\
& \rightarrow \min \left(h_{t}\left(m_{*}-1\right), h_{t}\left(m_{*}+1\right)\right), \quad u \rightarrow t .
\end{aligned}
$$

Therefore for $\gamma$ sufficiently large,

$$
\begin{aligned}
\sum_{m \neq m_{*}(t)} m^{k} \mathrm{e}^{-\gamma m} q_{m} & =\sum_{m \neq m_{*}(t)} \mathrm{e}^{-\gamma h_{k / \gamma}(m)} q_{m} \\
& \leq \mathrm{e}^{-\gamma \min \left(h_{k / \gamma}\left(m_{*}(t)-1\right), h_{k / \gamma}\left(m_{*}(t)+1\right)\right)} .
\end{aligned}
$$

It now follows from (4.8)-(4.10) that

$$
E\left(M^{k} \mathrm{e}^{-\gamma M}\right) \sim q_{m_{*}(t)}\left(m_{*}(t)\right)^{k} \mathrm{e}^{-m_{*}(t) \gamma},
$$

provided $q_{m_{*}}(t) \neq 0$, and, therefore, under assumption (4.7),

$$
R_{k}(\gamma) \rightarrow m_{*}(t)
$$

For $j=1,2, \ldots$, let

$$
a_{j}=\inf \left\{t \geq 0: m_{*}(t)=j\right\}=\frac{1}{\log j-\log (j-1)} .
$$

Then $a_{j}<a_{j+1}$, and by relation (4.11) we obtain the following result.

Proposition 4.6. For $j=1,2, \ldots$ let $t \in\left(a_{j}, a_{j+1}\right)$ be such that $q_{m_{*}}(t) \neq 0$. If $k$ and $\gamma$ grow according to (4.7), then

$$
R_{k}(\gamma) \rightarrow j
$$


That is, if $k$ and $\lambda$ are large, and $k / \lambda \in\left(a_{j}, a_{j+1}\right)$ for $j$ that is not very large, then $R_{k}(\gamma)$ will be close to $j$. Once again, this staircase-like behavior is neatly visible in Figure 4.5.

\section{Prediction in a nOn-life insurance data Set}

In this section we consider a non-life insurance data set which was kindly provided to us by Alois Gisler. The business line is not known to us. Our aim is to study the performance of our predictors on this data set, given suitable assumptions on the distributions $\left(q_{m}\right)$ and $\left(p_{j}\right)$ and the Poisson parameter $\mu$. Moreover, we will compare our predictors with those prescribed by the chain ladder method under Mack's conditions; see Section 1. We will focus on the prediction of the numbers of payments.

Our data contains claims that arrive in one year (1985) and the individual payment processes for each claim, including arrival date in 1985 and all dates and amounts of executed payments. Overall, 7,302 claims were incurred which triggered 24,606 payments through more than 10 years. Since we want to compare our method with the chain ladder prediction in Mack's framework, one of the problems we are facing is as follows. The chain ladder method requires a run-off triangle of data from different years; see (1.3). These data are needed for the construction of the chain ladder estimators of the factors $f_{j}$ in (1.2):

$$
\widehat{f_{j}}=\frac{\sum_{i=1}^{n-j-1} \sum_{r=0}^{j+1} N_{i, i+r}}{\sum_{i=1}^{n-j-1} \sum_{r=0}^{j} N_{i, i+r}},
$$

where $n$ is the number of years for which the run-off triangle is available. But we have only one year of claim arrivals at our disposal.

We solve this problem by switching from years to months. Then we have $n=12$ months of claim arrivals and the corresponding individual payment processes accounted for by months. Table 5.1 contains the monthly claim arrival numbers $M_{j}, j=1, \ldots, 12$, showing a clear seasonality in the data.

\begin{tabular}{|c|r|r|r|r|r|r|r|r|r|r|r|r|}
\hline$j$ & 1 & 2 & 3 & 4 & 5 & 6 & 7 & 8 & 9 & 10 & 11 & 12 \\
\hline$M_{j}$ & 414 & 391 & 493 & 552 & 641 & 765 & 748 & 762 & 741 & 635 & 628 & 532 \\
\hline
\end{tabular}

Table 5.1. The monthly claim numbers $M_{j}$

Table 5.2 contains the payment numbers in run-off triangle form. The $i$ th row contains the payment numbers $N_{i i}, \ldots, N_{i, 12}$ for claims arriving in the $i$ th month and whose payments are executed in month $i+k \in\{i, \ldots, 12\}$. These numbers form the input to the chain ladder method under Mack's procedure. In this case, the data set contains also the actual "future" monthly payment numbers $N_{i, 12+k}, k \geq 1$. We will try to predict these using both our method and the chain ladder method. We will construct both forecasts for each month of a whole year after the month $i, i=1, \ldots, 12$. The constraint on the prediction range is caused by the structure of the chain ladder method, but not of our procedure. Clearly, we do not predict the "observed" numbers 
in Table 5.2. The actual "future" numbers $N_{i, 13}, \ldots, N_{i, i+11}$ are in our data set, and they are presented in Table 5.3.

\begin{tabular}{r|rrrrrrrrrrrr} 
Month & 1 & 2 & 3 & 4 & 5 & 6 & 7 & 8 & 9 & 10 & 11 & 12 \\
\hline 1 & 40 & 147 & 141 & 120 & 99 & 84 & 67 & 63 & 42 & 44 & 47 & 35 \\
2 & & 40 & 144 & 132 & 100 & 85 & 71 & 53 & 63 & 52 & 50 & 25 \\
3 & & & 39 & 179 & 159 & 125 & 118 & 80 & 85 & 85 & 85 & 48 \\
4 & & & & 36 & 209 & 169 & 158 & 117 & 91 & 103 & 98 & 54 \\
5 & & & & & 60 & 217 & 224 & 159 & 136 & 125 & 110 & 86 \\
6 & & & & & & 75 & 280 & 229 & 186 & 191 & 145 & 121 \\
7 & & & & & & & 70 & 234 & 192 & 184 & 157 & 131 \\
8 & & & & & & & & 63 & 242 & 250 & 197 & 142 \\
9 & & & & & & & & & 62 & 246 & 227 & 195 \\
10 & & & & & & & & & & 64 & 200 & 175 \\
11 & & & & & & & & & & & 59 & 197 \\
12 & & & & & & & & & & & & \\
\end{tabular}

Table 5.2. The run-off triangle with the number of payments arising from 12 months of claim arrivals. The ith row contains the observed payment numbers $N_{i i}, \ldots, N_{i, 12}$.

\begin{tabular}{r|rrrrrrrrrrr} 
Month & 13 & 14 & 15 & 16 & 17 & 18 & 19 & 20 & 21 & 22 & 23 \\
\hline 1 & & & & & & & & & & & \\
2 & 33 & & & & & & & & & & \\
3 & 52 & 42 & & & & & & & & & \\
4 & 51 & 45 & 32 & & & & & & & & \\
5 & 68 & 53 & 56 & 42 & & & & & & & \\
6 & 107 & 79 & 68 & 75 & 55 & & & & & & \\
7 & 120 & 83 & 66 & 98 & 68 & 54 & & & & & \\
8 & 150 & 105 & 112 & 107 & 88 & 75 & 59 & & & & \\
9 & 148 & 140 & 115 & 131 & 107 & 85 & 80 & 56 & & & \\
10 & 174 & 118 & 122 & 103 & 82 & 77 & 76 & 57 & 45 & & \\
11 & 235 & 167 & 143 & 134 & 116 & 89 & 65 & 66 & 50 & 61 & \\
12 & 177 & 161 & 138 & 139 & 120 & 101 & 113 & 63 & 65 & 86 & 56
\end{tabular}

Table 5.3. The actual "future" payment numbers corresponding to the observations in Table 5.2. The ith row contains the monthly payment numbers $N_{i, 13}, \ldots, N_{i, i+11}$.

In our prediction procedure, we assume that the monthly claim numbers $M_{j}, j=1, \ldots, 12$, are Poisson distributed. Since there is a clear seasonality in the data we do not assume the $M_{j}$ 's identically distributed. Instead, we simply take each $M_{j}$ as the substitute of its expectation $E M_{j}$. Recall that this is the only parameter of the assumed Poisson distribution needed to compute the functions $R$ and, hence, to run our procedure; see Theorem 2.5. Clearly, the Poisson assumption on the distribution of $M_{j}$ 's is rather ad hoc, but we have only one data set, which makes it impossible to estimate the distributions of the $M_{j}$ 's. In general, such estimation has to be done on historical data.

On the other hand, the other ingredients needed for our procedure, the average Poisson number of payments per claim $\mu$ and the "displacement probabilities" $p_{j}$ in (1.4), can be easily estimated from our single data set. The estimate of the former is $\mu=3.37$, and the estimated the values of the $\left(p_{j}\right)$ are given in Table 5.4. Recall that we only need the values of $p_{j}$ with 
$j \leq 12$, which is what is presented in Table 5.4. In particular, the entries in the table do not add up to 1 .

\begin{tabular}{|c|r|r|r|r|r|r|r|r|r|r|r|r|}
\hline$j$ & 1 & 2 & 3 & 4 & 5 & 6 & 7 & 8 & 9 & 10 & 11 & 12 \\
\hline $100 \times p_{j}$ & 2.41 & 11.36 & 10.37 & 7.95 & 6.49 & 5.26 & 4.30 & 4.04 & 3.00 & 2.94 & 2.60 & 2.09 \\
\hline
\end{tabular}

Table 5.4. The estimated probabilities $p_{j}, j=1, \ldots, 12$.

In Table 5.5 we show the relative prediction error for our prediction procedure. The $i$ th row in this table shows the relative error in our prediction procedure of the values $N_{i, 12+k}, k=1, \ldots, i-1$, based on the values $N_{i i}, \ldots, N_{i, 12}$.

In the left column of Figure 5.7 we show the performance of our prediction procedure in two different situations. In the top graph we are in the situation of the 11th line of Tables 5.2 and 5.3. We predict the payments numbers for claims arriving in the 11th month. Two payment numbers have already been observed from which the payment numbers for months 13-22 get predicted (solid lines). The dots indicate the observed payment numbers and the bands around the predictions represent \pm 1.96 times the square of the conditional prediction error given in Theorem 2.5. In the bottom graph we are in the situation of the 7th line in Tables 5.2 and 5.3. We predict the payment numbers arising from claims arriving in the 7 th month. Six payment numbers have already been observed, and the payment numbers for months 13-18 are predicted.

Our predictions are compared with those prescribed by the chain ladder method under Mack's procedure (1.2). The multi-step prediction version of the latter is

$\widetilde{N}_{i, 12+k}=\left(f_{12-i+k-1}-1\right) f_{12-i+k-2} \cdots f_{12-i}\left(N_{i i}+\cdots+N_{i, 12}\right), k=1,2, \ldots$,

and then one replaces the $f$-factors by their chain ladder $\widehat{f}$-estimators given in (5.1).

In Table 5.6 we show the relative prediction error for the chain ladder predictor under Mack's procedure. Once again, the $i$ th row in this table shows the relative error in predicting the values $N_{i, 12+k}, k=1, \ldots, i-1$, based on the values $N_{i i}, \ldots, N_{i, 12}$. Note that a direct comparison of the relative errors in Tables 5.5 or 5.6 is difficult.

Similarly, in the right column of Figure 5.7 we show the chain ladder prediction for the same situations as with our predictor in the left column. Again, a direct comparison seems difficult. The error bands for the chain ladder method seem to be larger than for our method. Note that the bands around the predictions in the right column indicate \pm 1.96 times the square of the conditional prediction error given at the bottom of p. 363 in Mack [5]. These numbers are not model based and, consequently, their meaning is unclear.

It should be noted that, in practice, our procedure is likely to be used under far more favorable conditions than in the application presented in this section. A larger historical data set will allow for better estimation of the parameters required to run our forecasting procedure. Most importantly, in 


\begin{tabular}{r|rrrrrrrrrrr} 
Month & 13 & 14 & 15 & 16 & 17 & 18 & 19 & 20 & 21 & 22 & 23 \\
\hline 1 & & & & & & & & & & & \\
2 & -11.6 & & & & & & & & & \\
3 & -0.8 & 0.7 & & & & & & & & \\
4 & -29.8 & -33.0 & -48.9 & & & & & & & \\
5 & -32.9 & -56.1 & -33.6 & -41.9 & & & & & & \\
6 & -2.4 & -7.9 & -14.8 & 5.9 & -2.2 & & & & & & \\
7 & -1.8 & -36.5 & -33.5 & 17.7 & -7.3 & -7.6 & & & & \\
8 & 6.0 & -15.6 & -0.5 & 18.2 & 8.9 & 3.3 & 2.2 & & & \\
9 & 0.4 & 7.9 & 3.5 & 21.5 & 25.2 & 13.8 & 17.2 & 5.8 & & & \\
10 & 14.1 & -6.1 & 10.3 & 8.5 & -6.5 & 11.7 & 18.1 & 1.3 & 0.5 & & \\
11 & 17.1 & 3.2 & 5.4 & 11.7 & 12.2 & -6.1 & -13.0 & -1.9 & -21.6 & 20.6 & \\
12 & -4.0 & -6.1 & -2.7 & 14.7 & 13.6 & 11.6 & 26.7 & -2.2 & 9.3 & 38.0 & 24.2
\end{tabular}

Table 5.5. Relative prediction error in \% by our method of the values in Table 5.3 using the observations given in Table 5.2.

\begin{tabular}{r|rrrrrrrrrrr} 
Month & 13 & 14 & 15 & 16 & 17 & 18 & 19 & 20 & 21 & 22 & 23 \\
\hline 1 & & & & & & & & & & & \\
2 & 3.4 & & & & & & & & & \\
3 & 17.9 & 2.5 & & & & & & & & \\
4 & -13.3 & -6.5 & -28.4 & & & & & & & \\
5 & -13.8 & -22.0 & 0.7 & -18.7 & & & & & & \\
6 & -11.2 & -17.0 & -17.1 & 11.8 & -11.7 & & & & & \\
7 & 8.3 & -21.7 & -21.1 & 35.9 & 15.3 & -1.4 & & & & \\
8 & 14.3 & -10.5 & -0.2 & 20.8 & 15.2 & 20.1 & 1.7 & & & \\
9 & -6.9 & 7.3 & -1.4 & 17.4 & 21.5 & 11.9 & 28.8 & -2.9 & & & \\
10 & 14.3 & -8.3 & 15.4 & 9.0 & -9.3 & 7.9 & 23.6 & 13.3 & -3.7 & \\
11 & 28.0 & 9.5 & 11.0 & 26.6 & 22.6 & -1.6 & -9.0 & 7.2 & -0.7 & 30.4 & \\
12 & 67.5 & 68.0 & 73.3 & 106.5 & 117.1 & 104.4 & 139.1 & 68.9 & 10.21 & 227.0 & 129.2
\end{tabular}

Table 5.6. Relative prediction error in \% by the chain ladder method of the values in Table 5.3 using the observations given in Table 5.2.

the present situation we were unable to estimate the distribution of the number $M$ of the arriving claims, and had to postulate the Poisson distribution. Further, usage of months instead of years introduced seasonality in the data, which our procedure does not take into account. Even under these unfavorable conditions, the present study shows that our predictor performs no worse than the chain ladder prediction as regards closeness to the the actual payment numbers and magnitude (as well as reliability) of the conditional prediction errors.

For the three distributions of the $(a, b)$-class our prediction method is easily implemented by using standard software. When taking into account Remark 3.4, one gets quick numerical answers to the prediction problem. Notice that the distributions in the $(a, b)$-class are the most frequently used ones in applications. Our model requires knowledge of the probabilities $\left(q_{m}\right)$, $\left(p_{j}\right)$ and the Poisson parameter $\mu$. Once again, these quantities are easy to estimate if historical data are available.

We conclude that our prediction approach, which is based on a well defined stochastic model of claim arrivals and payment time distribution, works reasonably well in a "difficult" situation. Our model can be easily simulated from. Recall that the chain ladder estimator with Mack's procedure is not 
Prediction

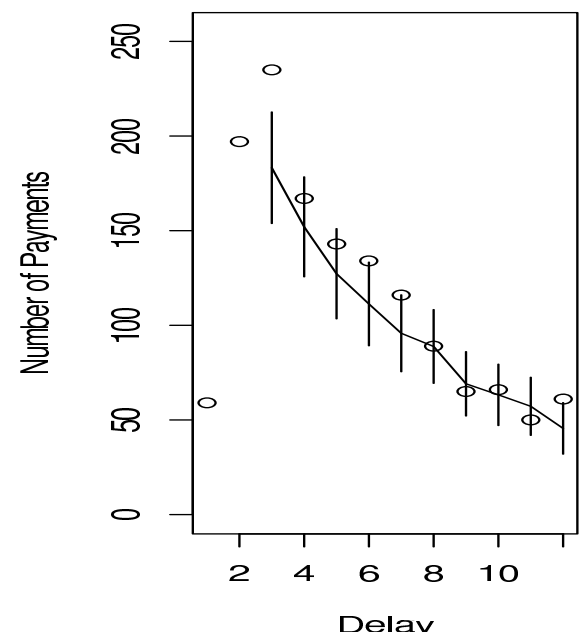

Prediction

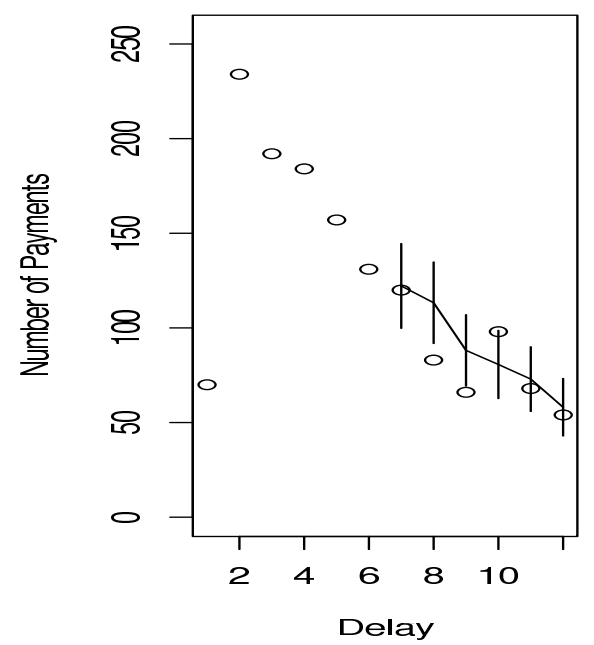

Chain ladder

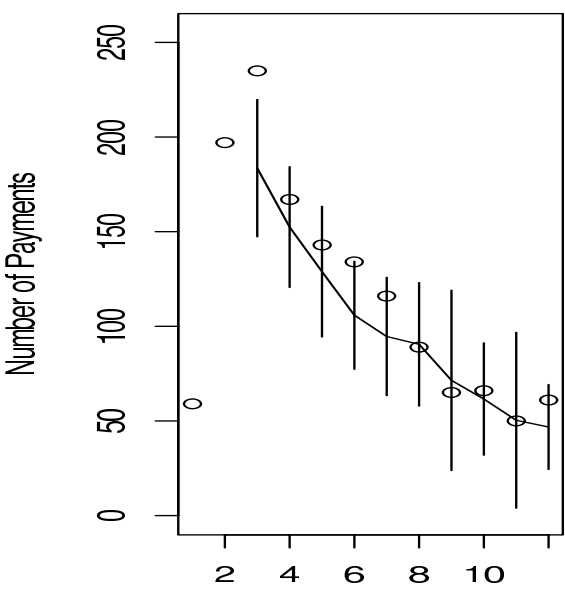

Delay

Chain ladder

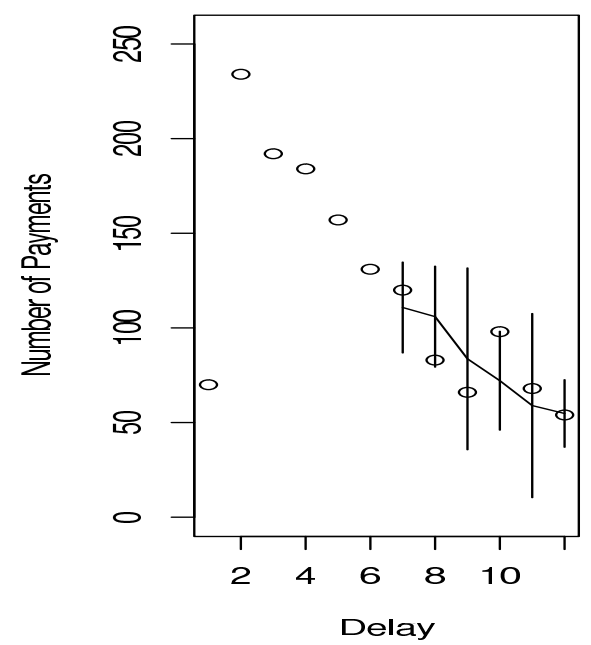

Figure 5.7. Predictions (solid lines) of monthly payment numbers by using the theory in Section 2.2 (left graphs) and the chain ladder approach (right graphs). The dots represent the payment numbers of the real-life data. The bands around the predictions represent \pm 1.96 times the square of the conditional prediction errors. The top graphs correspond to the 11 th row in Tables 5.2 and 5.3, the bottom ones to the 7th row.

model-based. Therefore, we consider it to be promising to use the suggested forecasting procedure under the dynamics in a non-life insurance portfolio.

\section{REFERENCES}

[1] Hess, K.T., Liewald, A. And Schmidt, K.D. (2002) An extension 
of Panjer's recursion. ASTIN Bulletin 32, 283-297.

[2] MACK, T. (1993) Distribution-free calculation of the standard error of chain ladder reserve estimates. ASTIN Bulletin 23, 213-225.

[3] MACK, T. (1994) Which stochastic model is underlying the chain ladder method? Insurance: Mathematics \&f Economics 15, 133-138.

[4] Mack, T. (1994) Measuring the variability of chain ladder reserve estimates. In: Proceedings of the Casualty Actuarial Society Forum, Spring 1994, Vol. 1, pp. 101-182.

[5] MACK, T. (1999) The standard error of chain ladder reserve estimates: recursive calculation and inclusion of a tail factor. ASTIN Bulletin $\mathbf{2 9}$, 361-366.

[6] Mack, T., Quarg, G. and Braun, C. (2006) The mean square error of prediction in the chain ladder reserving method. A comment. ASTIN Bulletin 36, 543-553.

[7] Mikosch, T. (2004) Non-Life Insurance Mathematics. An Introduction with Stochastic Processes. Springer, Heidelberg.

Department of Mathematics, University of Copenhagen, Universitetsparken 5, DK-2100 Copenhagen, Denmark

E-mail address: ahj@math.ku.dk

Department of Mathematics, University of Copenhagen, Universitetsparken

5, DK-2100 Copenhagen, Denmark

E-mail address: mikosch@math.ku.dk

School of Operations Research and Information Engineering, Cornell University, 220 Rhodes Hall, Ithaca, NY 14853, U.S.A.

E-mail address: gennady@orie.cornell.edu 